\title{
An attempt to develop an experimental data based model for annular section through EHD technique
}

\author{
S.K.Tahilyani ${ }^{1}$, Y.M.Jibhakate ${ }^{2}$ \\ ${ }^{\prime}$ (Heat Power, KITS, India) \\ ${ }^{2}$ (Heat Power, KITS, India)
}

\begin{abstract}
This paper describes the heat transfer enhancement through electro hydrodynamics technique (EHD). This is one of the active technique by which one can enhance the heat transfer rate. Keeping this as an epic centre the present work has been planned. An experimental set up is developed for simulating heat transfer phenomenon through EHD technique. Conversely the experimentation has been done for certain test points. Further using the observed experimental data a mathematical model has been formed and an analysis of experimental data based model is done.
\end{abstract}

Keywords: Electrohydrodynamics, intensification, corona, dielectric fluid, reliability, sensitivity

\section{Introduction}

Literature review indicates that heat transfer enhancement is an important phenomenon and it also indicates that heat transfer enhancement techniques are beneficial in increasing the performance of heat exchangers. There are three basic heat transfer modes: conduction, convection and radiation. Literature review indicates that heat transfer enhancement technique is broadly divided into two groups: active technique and passive technique. The active techniques require external forces, e.g. Electric field, acoustic or surface vibration etc. while the passive techniques require fluid additives or special surface geometries. EHD is one of the active techniques and has not yet been adopted commercially, largely because of concerns about installing the electrodes and using very high voltages during heat exchanger operation. It has a potential drawback, common to all active techniques, in that an extra system is required (in this case, the electrostatic generator); failure of this system means the enhancement is not obtained [10]. EHD heat transfer augmentation may yield large reductions in heat exchanger weight and volume, making this technique attractive for various engineering applications. As this technique operates electronically, it may be incorporated into the existing electronics of the thermal systems and conveniently controlled by computer. The controlling aspect of EHD is a clear advantage over the passive techniques that cannot be controlled electronically [12]. Over the past 70 years, the heat transfer enhancement by using strong electric field has been continuously studied [11]. But in many cases, however, analytical or numerical predictions of heat transfer and flow friction behavior are not yet possible and empirical correlations must be relied on for design. Modeling should be encouraged, however, because the resulting understanding of the phenomenon can result in better predictions and improved surface or insert device configurations [12]. In this regard an experimental set up is designed and fabricated. Using engineering experimentation and dimensional analyses Mathematical model for the particular design is developed.

\section{Background of present research}

The present work is pertaining to free convective heat transfer. Since the modern day heat transfer systems call for more efficient and compact exchangers to save energy and time, attempts are being made to achieve the same .Major emphasis is put on active methods of heat transfer enhancement as passive methods of heat transfer enhancement have certain limitations,. ElectroHydroDynamically enhanced heat transfer enhancement is one of the active technique. Heat transfer phenomenon itself is a very complex phenomenon for analysis. The entire phenomenon involves heat transfer material properties and the changes in it over a range of wide temperatures and pressures, the heat transfer medium and the changes in it over a range of wide temperatures and pressures, the geometry of the heat transfer surfaces and system and the other related changes in the dependent properties which will influence the heat transfer phenomenon and hence the heat transfer rate. Methodology of engineering experimentation applied to various experimental investigations is reported [8]. Such methodology can also be applied to experimental investigations for heat transfer processes. The equations governing the heat transfer process involving too many parameters both dependent and independent can be reduced using dimensional analysis [7]. With the help of experimental data recorded for the designed experimental set up, the effect of various parameters on the entire heat transfer phenomenon can be analyzed. The analysis of the effect of the parameters will give the dominance of the parameters affecting the heat transfer phenomenon. The modifications in the dominant parameters if possible can be done by trial and error employing the literature available about the work so far has been carried out to get the maximum possible heat transfer rate 
and hence maximum heat transfer. This approach can be followed to validate the dominance of parameters affecting the heat transfer phenomenon. In this regard an experimental set up is designed and fabricated. This will help in collecting data for experimental investigation. The dimensionless $\pi$ terms of dependent and independent variables are formed by applying theories of engineering experimentation which will help in representing the phenomenon. In experimentation voltage is varied and with the variation in voltage, variation in temperature of coil, surface of inner cylinder, dielectric fluid, outer surface and current is noted down. After noting down the readings change in other related parameters involved is calculated. So an experimental data is available now. This available data and the technique of multiple regression analyses are used to establish the mathematical model. The indices occurred for three independent $\pi$ terms involved in the dimensional equation which was derived to govern the heat transfer phenomenon, will indicate the dominance of particular parameter which will play relative roles in the heat transfer phenomenon.

\section{Objectives of the present research}

i. To affirm the impact on various parameters involved in heat transfer process by electric field

ii. To affirm the impact of all these parameters conjoin together on heat transfer rate

iii. By applying the theory of engineering experimentation and dimensional analyses an experimental data based model is constitute

iv. To analyze quantitatively the experimental data based model

\section{Design and fabrication of experimental setup}

The set up consist of two concentric cylinders made up of Beryllium Copper. A coil is placed inside the inner cylinder surrounded by moist plaster of Paris powder. Plaster of Paris powder is densely packed around the coil inside the inner cylinder. Inner cylinder is lidded with Bakelite (Electric insulator) to detach it electrical from the outer cylinder. Terminals of the coil are taken out of the lid. Inner surface of the outer cylinder is fitted with pins. These pins are placed radially around the cross section of the cylinder. These are equidistant around the circumferential and around the length of the cylinder. These pins are pointed in nature and will act as electrodes. Sharp points of the pins will help in giving rise to highly non uniform electrostatic fields. These electrostatic fields will augment heat transfer due to electrophoresis and lead to the generation of corona winds. Bottoms of both the cylinders are electrically detached by keeping height difference. Height of the inner cylinder is kept intentionally small to detach it electrically with outer cylinder. Top plate of the outer cylinder is cut from center with a diameter slightly larger than inner cylinder and the inner cylinder is suspended from top plate of the outer cylinder. Five calibrated thermocouples are attached to provide the temperature of coil, surface of inner and outer cylinder, fluid and atmosphere. This complete model is insulated from the surrounding by plaster of paris and with ropes in between to provide strength to the insulation after solidification. See Fig 1, 2, 3.

Following are the dimensions of the fabricated model: Diameter of the outer cylinder $\left(\mathrm{D}_{0}\right)$ is $0.2667 \mathrm{~m}$, diameter of the inner cylinder is $0.127 \mathrm{~m}$, height of outer cylinder is $0.35 \mathrm{~m}$, height of inner cylinder is 0.2985 $\mathrm{m}$, length of pin is $0.0635 \mathrm{~m}$, total number of pins used are 102, circumferential distance between the two consecutive pins is $0.0482 \mathrm{~m}$.

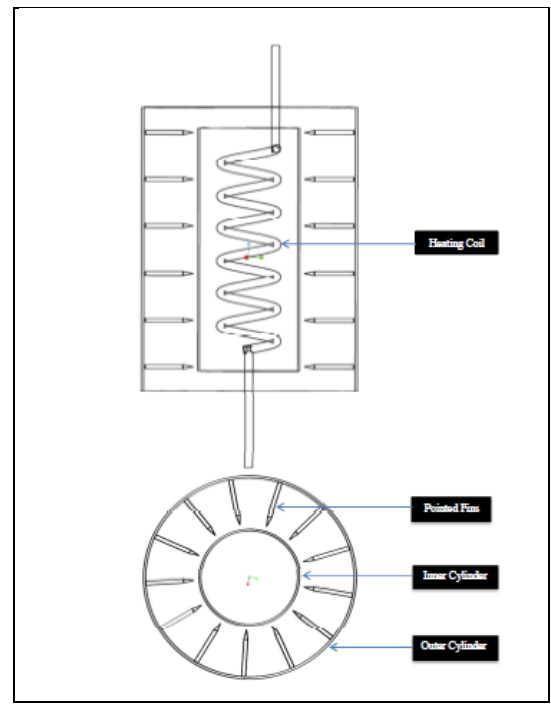

Fig 1: Experimental Set-Up 


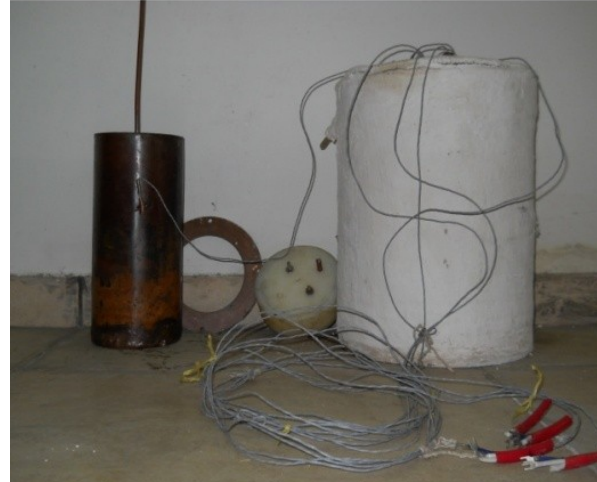

Fig 2: Image Showing Outer and Inner Cylinder

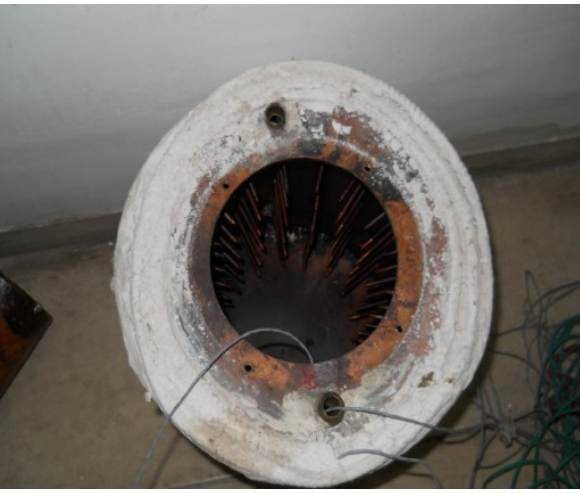

Fig 3 : Image Showing Pins fitted on the Inner Surface of Outer Cylinder

\section{Design of Experimentation}

Identification of various physical quantities which are going to affect the free convective heat transfer process is done followed by dimensional analyses. In this mass, length, time, temperature and dielectric constant are considered as fundamental quantities. The dimensional equation attained for the EHD heat transfer process is shown as below [8]:

$N u=\left\langle h D_{0} \mid K\right\rangle=\phi\left\langle\left(\frac{\mu C_{p}}{K}\right),\left(\frac{g \beta \rho \Delta T C_{p} D_{0} V^{2} \psi}{K^{2} T}\right),\left(\frac{D_{i}}{D_{0}}\right),\left(\frac{P}{D_{0}}\right),\left(\frac{S}{D_{0}}\right),\left(\frac{L}{D_{0}}\right)\right\rangle$

In equation (1), there is one dependent $\pi$ term and three independent $\pi$ terms. $\mathrm{Nu}=\left(\mathrm{hD}_{0} / \mathrm{K}\right)$ is the Nusselt number. It is the dependent $\pi$ term denoted by $\pi_{0}$. Whereas $\left(\frac{\mu C_{p}}{K}\right)$ is the prandtl number and is one of the independent $\pi$ term denoted by $\pi_{1},\left(\frac{g \beta \rho \Delta T C_{p} D_{0} V^{2} \psi}{K^{2} T}\right)$ is the modified Grashof number and also the second independent $\pi$ term denoted by $\pi_{2}$, Third independent $\pi$ term denoted by $\pi_{3}$ is $\left(\frac{D_{i}}{D_{0}}\right),\left(\frac{P}{D_{0}}\right),\left(\frac{S}{D_{0}}\right),\left(\frac{L}{D_{0}}\right)$ shows the geometrical parameters. For reducing the error to be commences while doing experimentation and also the outside influences and further to maximize the use of generated data test planning was planned. It involves three stages: Test envelope, Test Points, Test Sequence.

\section{Execution of experimentation}

Before performing experimentation the model was tested against leaks. Various safety measures were taken into account while performing the experimentation. Model was mounted on the wooden table so as to ensure electrical safety while experimentation. Set up transformer and measuring instruments were kept quite apart from the model to ensure safety while operating and noting data. Thermocouples those are mounted on the model, fitted with larger length wires.

The first step for experimentation was to heat the coil. This was done by supplying electricity to the coil. Basic reason for this is to transfer heat from coil to surface of inner cylinder. Plaster of paris will help in maintaining the constant heat flux around the surface of inner cylinder and as it is having high thermal conductivity it will help in maintaining the constant temperature for a sufficient duration of time. It takes duration of 1 hour or 2 hours or more to achieve the steady flux condition and once this condition is achieved high $\mathrm{AC}$ voltage was applied on the inner cylinder and on the tips of the pins. This leads to the corona winds generation. As discussed above, gradually voltage was increased and the corresponding readings of temperatures and current was noted down,

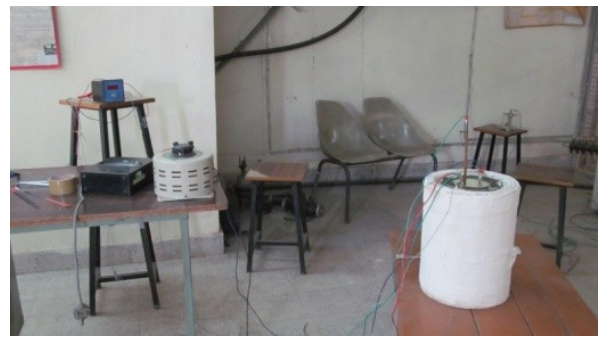

Fig 4 : Image of Experimental Set-Up with Connections 
In this experimentation three dielectric fluids Air, Transformer oil (unused) and Glycerin are used. As thermal properties of Air are readily available it was selected as one of the dielectric fluid. Test points selected for experimentation was from $230 \mathrm{~V}$ to $1030 \mathrm{~V}$. Intervals were kept at a difference of $200 \mathrm{~V}$. A Common procedure for experimentation and common test points were decided for the three dielectric fluids. Temperature difference $\Delta \mathrm{T}$ between the inner cylinder and fluid was decided to maintained approximately $50 \mathrm{~K}$. First readings for Air were noted down. Then the model was filled with transformer oil from the holes made on the top of the top plate of outer cylinder. After filling the fluid these holes were further screw tied to avoid leakages. Again the same procedure was followed as that for air and readings were noted down. Transformer oil is then evacuated out by taking out the inner cylinder. Then it was cleaned with the help of kerosene and kept under sunlight to dry.

Then experimentation was performed again for Glycerin.

Heat influx to the system,

$\mathrm{Q}=\mathrm{h} \mathrm{A} \Delta \mathrm{T}$

Heat influx to the system can also given as,

$\mathrm{Q}=\mathrm{V} * \mathrm{I}$

From equation (2) and (3)

$\mathrm{Q}=\mathrm{h} \mathrm{A} \Delta \mathrm{T}=\mathrm{V} * \mathrm{I}$

From equation (4)

$\mathrm{h}=\mathrm{V} * \mathrm{I} / \mathrm{A} * \Delta \mathrm{T}$

Heat transfer coefficient $\mathrm{h}$ can be calculated from equation (5).

\section{Observations}

While experimentation it was observed that for reaching the steady heat flux one hour or more time is required. It was difficult to maintain the temperature difference of approximately $50 \mathrm{k}$. As higher voltage was applied to the inner cylinder and the pointed pins a significant change in the temperature of dielectric fluid was noticed and no change in the temperature of the coil. So the current to the coil has to be adjusted to maintain a temperature difference of $50 \mathrm{~K}$. And one this steady condition is achieved observations was noted down.

It was observed that there is a significant increase in heat transfer coefficient in all the three fluids with the increase in voltage.

\section{Formulation of experimental data based model}

In Schenck H.Jr [8] an approach for formulation of experimental data based model is proposed. This approach is adopted in modified form. This modification is to the range of enclosing only independent variables and recorded data. This is accomplished by reducing the number of independent variables through dimensional analysis, collecting the experimental data of heat transfer intensification process directly and by formation of the experimental data based model. For this particular model having peculiar geometry described above the heat transfer enrichment through EHD technique is investigated. An attempt is made to establish an experimental data based model for this particular geometry .For this variation in fluid properties and temperatures across heat transfer surfaces are consider.

Dimensional equation arrived through reduction of variables is shown in equation (1). Formulation of model is achieved by recognizing the constant and indices of $\pi$ terms. By acknowledging the independent and dependent $\pi$ terms, the multiple regression analyses helps to recognize the indices of $\pi$ terms in the model aimed at. Let the model be in the form,

$$
\left(\pi_{\mathrm{o}}\right)=\mathrm{K} *\left\{\left(\pi_{1}\right)^{\mathrm{a}} *\left(\pi_{2}\right)^{\mathrm{b}} *\left(\pi_{3}\right)^{\mathrm{c}}\right\}
$$

From equation (1),

If

$\left(\mathrm{h} \mathrm{D}_{0} / \mathrm{K}\right)=\mathrm{Y}$;

$\left.\left(\mu \mathrm{C}_{\mathrm{p}}\right) / \mathrm{K}\right)=\mathrm{A}$;

$\left(\mathrm{g} \beta \rho \Delta \mathrm{T} \mathrm{C}_{\mathrm{p}} \mathrm{D}_{0} \mathrm{~V}^{\wedge} 2 \psi\right) /\left(\mathrm{K}^{\wedge} 2 \mathrm{~T}\right)=\mathrm{B}$ and

$\left(\mathrm{D}_{\mathrm{i}} / \mathrm{D}_{0}\right),\left(\mathrm{P} / \mathrm{D}_{0}\right),\left(\mathrm{S} / \mathrm{D}_{0}\right),\left(\mathrm{L} / \mathrm{D}_{0}\right)=\left(\mathrm{D}_{\mathrm{i}} \mathrm{S} / \mathrm{PL}\right)$

Then regression equations formed are :

$\Sigma \mathrm{Y}=\mathrm{nK}_{1}+\mathrm{a} \Sigma \mathrm{A}+\mathrm{b} \Sigma \mathrm{B}+\mathrm{c} \Sigma \mathrm{C}$

$\Sigma \mathrm{Y}=14 \mathrm{~K}_{1}+\mathrm{a} \Sigma \mathrm{A}+\mathrm{b} \Sigma \mathrm{B}+\mathrm{c} \Sigma \mathrm{C}$

$\Sigma \mathrm{YA}=\mathrm{K}_{1} \Sigma \mathrm{A}+\mathrm{a} \Sigma \mathrm{A}^{2}+\mathrm{b} \Sigma \mathrm{AB}+\mathrm{c} \Sigma \mathrm{AC}$

$\Sigma \mathrm{YB}=\mathrm{K}_{1} \Sigma \mathrm{B}+\mathrm{a} \Sigma \mathrm{AB}+\mathrm{b} \Sigma \mathrm{B}^{2}+\mathrm{c} \Sigma \mathrm{BC}$

$\Sigma \mathrm{YC}=\mathrm{K}_{1} \Sigma \mathrm{C}+\mathrm{a} \Sigma \mathrm{AC}+\mathrm{b} \Sigma \mathrm{BC}+\mathrm{c} \Sigma \mathrm{C}^{2}$

Where,

$\mathrm{n}$ is number of set of readings

$\mathrm{A}, \mathrm{B}$ and $\mathrm{C}$ represents $\pi_{1}, \pi_{2}, \pi_{3}$ 
Y represents $\pi_{0}$

Now by solving the matrix in MATLAB software, the values of indices and constant are achieved.

$\mathrm{K}=2.2630805, \mathrm{a}=0.0026, \mathrm{~b}=0.0090, \mathrm{c}=-1.9902$

Substituting above achieved values in equation (6) we get,

$\left(\pi_{\mathrm{o}}\right)=2.2630805 *\left\{\left(\pi_{1}\right)^{0.0026} *\left(\pi_{2}\right)^{0.0090} *\left(\pi_{3}\right)^{-1.9902}\right\}$

So equation (1) will turn as under

$\mathrm{Nu}=\left\langle h D_{0} / K\right\rangle=2.2630805 *\left\langle\left(\mu C_{p} / K\right)^{0.0026} *\left(\frac{g \beta \rho \Delta T C_{p} D_{0} V^{2} \psi}{K^{2} T}\right)^{0.0090} *(\mathrm{DiS} / \mathrm{PL})^{-1.9902}\right\rangle$

\section{Quantitative analyses of the model}

Quantitative analyses of the model established in equation 13 is followed in two steps

i. By checking the reliability of the model

ii. By sensitivity analyses

Reliability of the model is estimated by substituting the known values of independent variables in the model obtained in equation (13) and values of dependent variables is obtained which will be known as calculated values of dependent variables. Now error can be found in the calculated value of dependent variables and observed values of dependent variables. This is done by subtracting the calculated value from the observed value of dependent variable. Further reliability of the model will be calculated by calculating the mean error. Reliability of the model obtained is $78.6564 \%$

After reliability sensitivity of the model is evaluated. This is done to evaluate the sensitiveness of individual $\pi$ term. The purpose of sensitivity check is to check the influence of individual independent $\pi$ term on dependent $\pi$ term. This is achieved by introducing $\pm 10 \%$ change in individual $\pi$ terms independently and the respective change in dependent $\pi$ term is obtained. One thing can be noticed that a total rate of change introduced in individual independent $\pi$ is $20 \%$. So the percentage change in dependent $\pi$ term obtained by introducing $20 \%$ change in individual $\pi$ term is shown graphically in fig (5) :

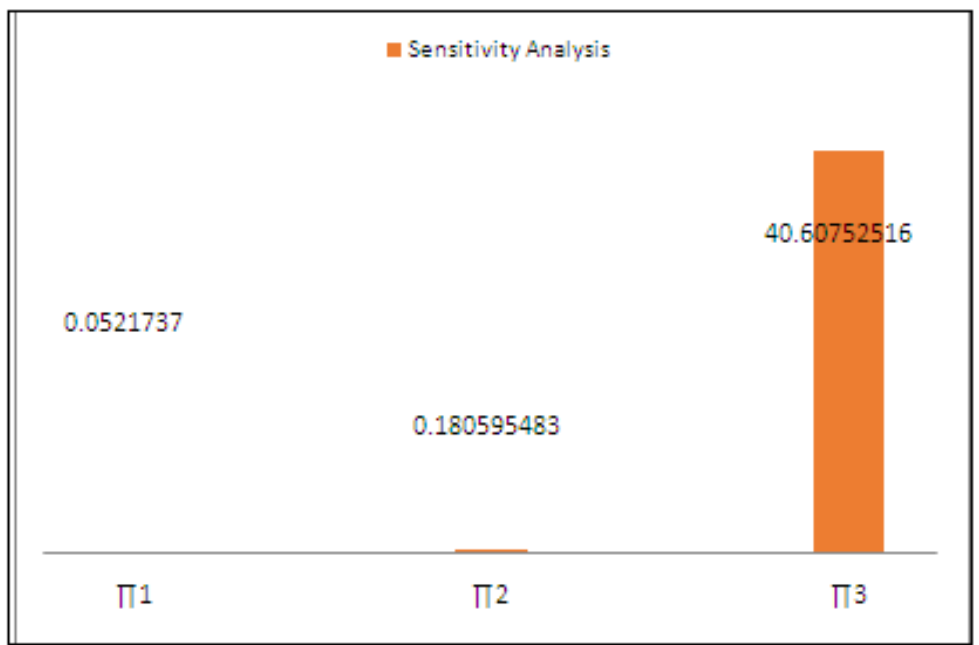

Fig 5 : Graphical Representation of Sensitivity Analysis(Percentage change in dependent variable)

\section{Conclusion}

i. It is concluded that, absolute index of $\pi_{2}$ is the highest index of $\pi_{0}$ and the absolute index of $\pi_{3}$ is the lowest index of $\pi_{0}$

ii. So the modified Grashoffs number influences the most and geometrical parameters influence the least to Nusselts number.

iii. So all the parameters of modified Grashoffs number will influence the Nusselts number. Parameters in the numerator are directly related to the Nuselts number and parameters in the denominator are inversely related to it.

iv. The value computed from the product of the various terms of the model will be magnified by constant $\mathrm{K}$ as its value is more than 1 . 


\section{Future Scope}

Range of values of applied voltage can be increased it is limited in the present research due to lack of availability and finance.

\section{References}

\section{Journal Papers:}

[1] A. Shoostari, M. Ohadi and F. H.R. Franca, Experimental and Numerical Analysis of Electrohydrodynamic Enhancement of Heat transfer in Air Laminar Channel Flow, IEEE SEMI - THERM Symposium, 19, 2003, 48-52.

[2] J. Darabi, , M. M. Ohadi, and D. DeVoe, An Electrohydrodynamic Polarization Micropump for Electronic Cooling, Journal of Microelectromechanical Systems, 10, 2001, 98-106.

[3] J.S.Cotton, J.S.Chang, M. Shoukri, T. Smith-Pollard, Electrohydrodynamically enhanced flow boiling in an eccentric horizontal cylindrival channel, Annual Report Conference on Electrical Insulation and Dielectric Phenomena, 2002.

[4] J.S.Paschkewitz, D.M.Pratt, The influence of fluid properties on Electrohydrodynamic Heat Transfer Enhancement in liquids under viscous and electrically dominated flow conditions, Experimental Thermal Fluid Science, 21, 187-97.

[5] T.V.V.R. Apparao, Ye-di Liu, N.V.Suryanarayana, Heat Transfer Augmentation in an Annulus with Interrupted Axial Fins, International Heat Transfer Conference, General Papers, Jerusalem, Israel, 9(4), 1990, 27-31.

[6] K. T. Tan, F. C. Lai, EHD enhanced Natural Convection In an enclosure : Effects of non-symmetric Electric field, Proc. of National Heat Transfer Conference, ASME, 2001.

[7] J. P. Modak, A. M. Pingle, Application of methodology of Engineering Experimentation to Investigation of Heat Transfer Augmentation Process Employing EHD Technique, National HMT Conference, 6, 229-232.

\section{Books:}

[8] Schneck,H, Theories of Engg. Experimentation ( 1961).

\section{Journal Papers:}

[9] A. E. Bergles, Some perspectives on enhanced heat transfer - second-generation heat transfer technology, ASME Journal of Heat Transfer, 110, 1988, 1082-1096.

[10] A. E. Bergles, Heat transfer Enhancement - The encouragement and accommodation of high heat fluxes, ASME Journal of Heat Transfer ,119, 1997, 8-19.

[11] L. P. Suryan., A review on electrohydrodynamic enhancement of heat transfer, JGSEE, 1996, 859-872.

[12] M. Molki and P. Damronglerd, Electrohydrodynamic Enhancement of Heat Transfer for Developing Air Flow in Square Ducts, Heat Transfer Engineering, 27(1), 2006, 35-45. 